\title{
Relaxation phenomena at criticality
}

\author{
A. Gambassi ${ }^{1,2, a}$ \\ 1 Max-Planck-Institut für Metallforschung, Heisenbergstr. 3, 70569 Stuttgart, Germany \\ 2 Institut für Theoretische und Angewandte Physik, Universität Stuttgart, Pfaffenwaldring 57, 70569 Stuttgart, Germany
}

Received 14 September 2007

Published online 1st February 2008 - (c) EDP Sciences, Società Italiana di Fisica, Springer-Verlag 2008

\begin{abstract}
The collective behaviour of statistical systems close to critical points is characterized by an extremely slow dynamics which, in the thermodynamic limit, eventually prevents them from relaxing to an equilibrium state after a change in the thermodynamic control parameters. The non-equilibrium evolution following this change displays some of the features typically observed in glassy materials, such as ageing, and it can be monitored via dynamic susceptibilities and correlation functions of the order parameter, the scaling behaviour of which is characterized by universal exponents, scaling functions, and amplitude ratios. This universality allows one to calculate these quantities in suitable simplified models and field-theoretical methods are a natural and viable approach for this analysis. In addition, if a statistical system is spatially confined, universal Casimir-like forces acting on the confining surfaces emerge and they build up in time when the temperature of the system is tuned to its critical value. We review here some of the theoretical results that have been obtained in recent years for universal quantities, such as the fluctuation-dissipation ratio, associated with the non-equilibrium critical dynamics, with particular focus on the Ising model with Glauber dynamics in the bulk. The non-equilibrium dynamics of the Casimir force acting in a film is discussed within the Gaussian model.
\end{abstract}

PACS. 64.60.Ht Dynamic critical phenomena - 64.60.an Finite-size systems

\section{Introduction}

Critical points occur in the phase diagrams of a variety of microscopically different systems, ranging from magnetic materials (ferromagnetic/paramagnetic transition), to pure fluids (liquid/vapour critical point), binary mixtures (mixing/demixing transition) and strongly interacting matter (QCD). In spite of these differences at the microscopic scale, a unified picture of the collective critical behaviour emerges in terms of the so-called order parameter $\varphi$ of the transition, the nature of which depends on the specific system while its fluctuations determine the physical behaviour close to critical points. In particular, in the case of ferromagnetic materials, $\varphi(\mathbf{x}, t)$ can be identified with the coarse-grained magnetization density at point $\mathbf{x}$ and time $t$, whereas for a fluid $A / B$ binary mixture with critical concentrations $c_{A}^{\text {crit }} / c_{B}^{\text {crit }}, \varphi$ is given alternatively, by $c_{A}-c_{A}^{\text {crit }}$ or $c_{B}-c_{B}^{\text {crit }}$ where $c_{A, B}$ are the space-time dependent concentrations of the species $A$ and $B$, respectively. The thermal fluctuations of the order parameter field $\varphi$ are correlated in space and time across a typical correlation length $\xi$ and a (linear) relaxation time $t_{R}$. Upon approaching the critical point, $\xi$ and $t_{R}$

\footnotetext{
a e-mail: gambassi@mf.mpg.de
}

become much larger than the corresponding microscopic length and time scales $\ell_{\text {micr }}$ and $\tau_{\text {micr }}$, respectively, resulting in a spatially collective and temporally slow behaviour which is characterized by a certain degree of universality. In fact, the physics at scales much larger than $\tau_{\text {micr }}$ and $\ell_{\text {micr }}$ becomes largely independent of the microscopic details of the system, depending only on its gross features such as the range of the microscopic interactions, internal symmetries, spatial dimensionality $d$, conservation laws etc. which characterize the so-called universality class of the transition. Universality is primarily an experimental fact based on the evidence that, e.g., $\xi \sim \xi_{0}|r|^{-\nu}$ and $t_{R} \sim \tau_{0}|r|^{-\nu z}$ for $r \equiv\left(T-T_{c}\right) / T_{c} \rightarrow 0$, where $T_{c}$ is the critical temperature, $\xi_{0}$ and $\tau_{0}$ specifically depend on the microscopic details of the system and therefore are nonuniversal, whereas $\nu$ and $z$ are universal exponents in the sense specified above $[1,2]$. Universality can be exploited in order to study the critical behaviour of a statistical system via suitable minimal models having the same gross features as the actual system and therefore belonging to the same universality class. In most of the cases this is done in terms of effective theories for the order parameter field $\varphi(\mathbf{x}, t)$ and the resulting critical scaling properties can be analyzed by means of powerful field-theoretical and renormalization-group methods [1]. 


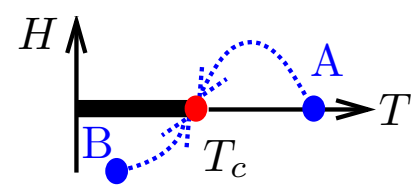

Fig. 1. Quenches in the phase diagram of an Ising ferromagnet: $T$ is the temperature of the bath, $H$ the external magnetic field and $(T, H)=\left(T_{c}, 0\right)$ is the critical point. The initial conditions $\mathrm{A}$ and $\mathrm{B}$ are characterized by a vanishing and non-vanishing magnetization, respectively.

The slow and collective behaviour which emerges close to critical points affects relaxation phenomena and the way in which they actually occur. In Section 2 we discuss the case of bulk systems, with particular emphasis on the emerging non-equilibrium properties, whereas in Section 3 we focus on confined critical systems in film geometry.

\section{Non-equilibrium relaxation in the bulk}

Altough the picture presented below is quite general, we shall refer specifically to the case of uniaxial ferromagnets belonging to the Ising universality class, with a critical temperature $T_{c}$ and a space-time dependent order parameter $\varphi(\mathbf{x}, t)$ (magnetization density).

One of the simplest instances of relaxation phenomena can be observed after a sudden thermal perturbation. In fact, assume that a system, initially in equilibrium with a thermal bath at temperature $T_{0}$, is brought at time $t=0$ in contact with a thermal bath at temperature $T \neq T_{0}$. As a consequence of this perturbation the system starts evolving out of equilibrium in a way which is expected to depend on the specific initial condition (e.g., on $T_{0}$ ). After this transient regime, of typical duration $t_{\mathrm{EQ}}(T)$, the system equilibrates with the thermal bath at temperature $T$, loses memory of the thermal quench and its dynamics becomes stationary and invariant (in the absence of external fields) under time reversal; fluctuations are therefore described by equilibrium dynamics. The duration $t_{\mathrm{EQ}}(T)$ of the transient regime, i.e., of the relaxation, depends on the temperature $T$ and is such that $t_{\mathrm{EQ}}\left(T>T_{c}\right)$ is finite whereas $t_{\mathrm{EQ}}\left(T \leq T_{c}\right)$ is actually infinite (in the thermodynamic limit) and the non-equilibrium relaxation goes on forever. In particular, we focus here on the case of a quench to the critical point $T=T_{c}$, referring the reader to reference [3] for a discussion of the phase ordering dynamics for $T<T_{c}$. The fact that $t_{\mathrm{EQ}}\left(T=T_{c}\right)$ is not finite is actually due to the emergent slow and collective critical behaviour. In this context it is important to understand the general features of this non-equilibrium relaxation, exploring, e.g., how it depends on the initial condition of the system. In particular - referring to Ising-like magnets - we will consider the cases depicted in Figure 1 of quenches from an initial state with vanishing $(\mathrm{A})$ and non-vanishing (B) magnetization. In addition to the dependence on the initial condition, it is also of interest to understand how the universality which characterizes critical phenomena manifests itself in the long-time relaxation

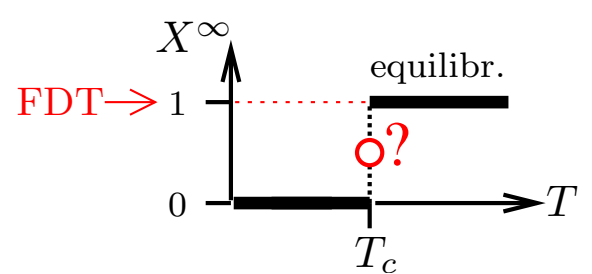

Fig. 2. $X^{\infty}$ as a function of the final temperature $T$ of the quench starting from a disordered initial state [9]. The value of $X^{\infty}$ for $T=T_{c}$ depends on the universality class of the system.

and if a scaling behaviour actually emerges. Some of these issues were already addressed more than 30 years ago, with particular focus on the dynamics of the order parameter $[4,5]$. Only quite recently, however, it has been realized [6] that dynamic correlations and susceptibilities display very interesting non-equilibrium properties such as those observed in glassy systems, for example ageing (see, e.g., Ref. [7] for a review). In this respect, critical dynamics provides a valuable, non-trivial but relatively simple instance of dynamics with ageing, which can be characterized to an extent actually out of reach in more general cases of glassy dynamics.

\subsection{Dynamic observables}

The relaxation process in a system of large volume $V$ can be monitored by looking at the global order parameter $\Phi(t)=V^{-1} \sum_{x \in V} \varphi(x, t)$, its average over the possible realization of the thermal noise $M(t)=\langle\Phi(t)\rangle$ and its two-time connected correlation function $C(t, s)=$

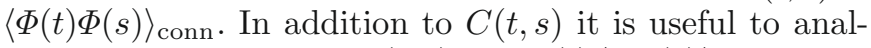
yse the linear response $R(t, s)=\delta M(t) /\left.\delta H(s)\right|_{H=0}$ of the global order parameter $M(t)$ to a magnetic perturbation $H(s)$ applied at time $s<t$. With the aim of highlighting non-equilibrium behaviours, we consider the so-called fluctuation-dissipation ratio (originally introduced in the study of glasses $[6,8]$ )

$$
X(t, s) \equiv \frac{T R(t, s)}{\partial_{s} C(t, s)},
$$

and in particular its limit for well-separated times

$$
X^{\infty} \equiv \lim _{t \rightarrow \infty} \lim _{s \rightarrow \infty} X(t, s)
$$

Indeed, for $t, s \gg t_{\mathrm{EQ}}(T)$ the fluctuation-dissipation theorem (see, e.g., Ref. [7]) implies $X(t, s)=1$ and therefore $X^{\infty}=1$ whenever equilibrium is eventually attained, which is always the case for $T>T_{c} ; X^{\infty} \neq 1$, instead, signals that the system evolves out of equilibrium even at long times. In particular, for quenches from the disordered initial state $(M(t<0)=0)$ to $T<T_{c}$ and $H=0$ (domain coarsening), scaling arguments yield $X^{\infty}=0$, leading to the global picture in Figure 2 [9]. The value of $X^{\infty}$ for $T=T_{c}$, on the other hand, cannot be fixed on the basis of general arguments but it depends on the specific system (actually on the universality class which it belongs to [9]). 
Note that, in contrast to the case of the non-equilibrium dynamics for $T<T_{c}$ (phase ordering) in which thermal fluctuations do not affect the leading scaling behaviour [3], the dynamics at $T=T_{c}$ is actually driven by them and by the emerging collective behaviour of the system. In recent years $X^{\infty}$ has attracted a lot of attention in connection to the fact that, formally, it might be used to define an effective non-equilibrium temperature $T_{\text {eff }}$ for the system (see, e.g., Ref. [7] and references therein). Indeed, at large times, the rhs of equation (1) equals 1 (as required by the equilibrium fluctuation-dissipation theorem) if one assumes that the temperature of the system is not given by the temperature $T$ of the bath but by $T_{\text {eff }}=T / X^{\infty}$. In the case of mean-field glassy models it has been shown that $T_{\text {eff }}$ is actually a bona fide thermodynamic temperature which controls, e.g., the direction of heat flows [7]. In this context, the non-equilibrium critical relaxation provides a simple instance of slow dynamics in which such a property can be tested beyond mean field [10].

To give answers to the various questions posed so far, it is possible to fully exploit universality $[9,11]$ in order to characterize within a field-theoretical approach $[11,12]$ the non-equilibrium critical relaxation and the associated ageing phenomena (see Ref. [13] for a pedagogical introduction). This approach allows a systematic analysis of several aspects of these relaxation phenomena and yields analytic predictions for scaling functions, exponents, and amplitude ratios which characterize the scaling behaviour of correlation and response functions at large times, within different universality classes and dynamics. In addition, it is possible to highlight dynamical crossovers in $C(t, s), R(t, s)[14,15]$ and in the persistence properties of $M(t)[16]$, due to different initial conditions. Within this field-theoretical approach, for example, instead of studying on a lattice $\mathbb{Z}^{d}$ the non-equilibrium dynamics of the Ising model with spin-flip Glauber dynamics (which captures the behaviour of some anisotropic ferromagnets and alloys), one studies the Landau-Ginzburg effective Hamiltonian $\mathcal{H}[\varphi][1]$ for the order parameter $\varphi(\mathbf{x}, t)\left(\mathbf{x} \in \mathbb{R}^{d}\right)$ with a suitable relaxational dynamics, known as Model A [2]:

$$
\partial_{t} \varphi(\mathbf{x}, t)=-D \frac{\delta \mathcal{H}[\varphi]}{\delta \varphi(\mathbf{x}, t)}+\zeta(\mathbf{x}, t)
$$

$D$ is a kinetic coefficient and $\zeta(\mathbf{x}, t)$ a zero-mean stochastic Gaussian noise with $\left\langle\zeta(\mathbf{x}, t) \zeta\left(\mathbf{x}^{\prime}, t^{\prime}\right)\right\rangle=2 k_{\mathrm{B}} T D \delta(\mathbf{x}-$ $\left.\mathbf{x}^{\prime}\right) \delta\left(t-t^{\prime}\right)$. The results presented below refer to this case.

\subsection{Scaling behaviour}

The renormalization-group analysis of Model A predicts that $R(t, s)$ and $C(t, s)$ display the following scaling behaviours after a quench to $T=T_{c}[17]$ :

$$
s<t, \quad\left\{\begin{array}{l}
R(t, s)=A_{R} t^{a}\left(\frac{t}{s}\right)^{\theta} \mathcal{F}_{R}\left(s / t, t / t_{0}\right), \\
C(t, s)=A_{C} t^{a+1}\left(\frac{t}{s}\right)^{\theta-1} \mathcal{F}_{C}\left(s / t, t / t_{0}\right),
\end{array}\right.
$$

where $a=(2-\eta-z) / z$ is given in terms of well-known universal equilibrium static $(\eta)$ and dynamic $(z)$ exponents [1], $\theta$ is the non-equilibrium universal initial-slip exponent [17], $\mathcal{F}_{R, C}$ are universal scaling functions normalized such that $\mathcal{F}_{R, C}(0,0)=1 . A_{R, C}$ are non-universal constants whereas $t_{0}$ is a non-universal time scale set by the initial value of the magnetization $M_{0} \equiv M(t=0)$ and which displays a universal dependence on it

$$
t_{0}=B_{m} M_{0}^{-1 / \kappa}
$$

where the universal scaling exponent $\kappa>0$ is given, in terms of static and dynamic equilibrium and non-equilibrium exponents, by $\kappa=\theta+a+\beta /(\nu z)$. The non-universal amplitude $B_{m}$ can be fixed by suitable normalization conditions (see Refs. [14,17] for details).

Interestingly enough, equations (4) and (5) indicate that of the initial condition only the value $M_{0}$ of the magnetization really matters in determining the scaling properties of the ensuing relaxation. A more detailed analysis shows that correlations in the initial state (as long as they are short ranged) do only contribute to corrections to the scaling behaviour [17]. According to equation (4), the twotime quantities $C(t, s)$ and $R(t, s)$ are homogeneous functions of the three time scales $t, s$, and $t_{0}$. In particular, when $s<t \ll t_{0}$, which is always the case if $M_{0}=0$, the scaling form of $R$ (analogous one for $C$ ) becomes

$$
R\left(t \ll t_{0}, s\right)=A_{R} t^{a}(t / s)^{\theta} f_{R}^{(0)}(s / t),
$$

where $f_{R}^{(0)}(x)=\mathcal{F}_{R}(x, 0)$. Equation (6) clearly displays the scaling behaviour typical of ageing phenomena: As a function of the time $t$ at which the effect of the magnetic perturbation is measured, the relaxation time is set by the time $s$ at which the perturbation was applied, which is also referred to as the age of the system, being the time elapsed since the quench. Upon increasing $s$ the "response" to the magnetic perturbation becomes increasingly slow. The scaling behaviour in equation (6) was already spelled out in reference [17] even tough the connection with ageing has been realized some years later [6]. If $M_{0}=0$ the scaling in equation (6) (and analogous one for $C$ ) is valid also at long times and allows one to express $X^{\infty}$ as an amplitude ratio $X^{\infty}=A_{R} /\left[(1-\theta) A_{C}\right][9,11]$. This amplitude ratio turns out to be universal and therefore its value calculated within the field-theoretical approach can be compared with the corresponding results obtained on the basis of different models in the same universality class (e.g., Ising model with Glauber dynamics studied via Monte Carlo simulations).

In the opposite limit of large times compared to $t_{0}$, i.e., $t_{0} \ll s<t$ the scaling form of $R$ (analogous one for $C)$ becomes [14]:

$$
R\left(t, s \gg t_{0}\right)=a_{R} t^{a}(t / s)^{-\beta \delta /(\nu z)} f_{R}^{(\infty)}(s / t),
$$

where $f_{R}^{(\infty)}(x) \sim x^{\theta+\beta \delta /(\nu z)} \mathcal{F}_{R}(x, y \rightarrow \infty)$ and $a_{R}$ is determined such that $f_{R}^{(\infty)}(0)=1$. Equation (7) displays a scaling behaviour (with ageing) analogous to the one 


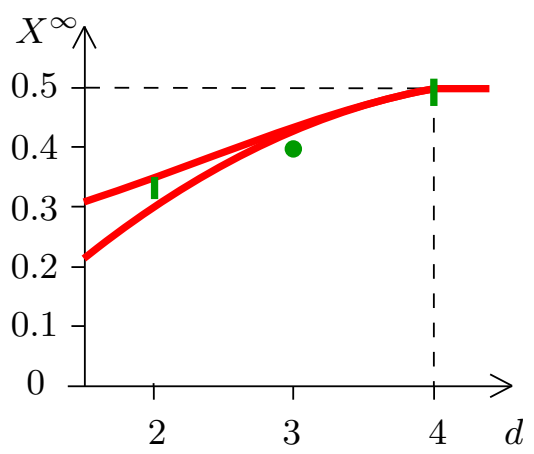

(a)

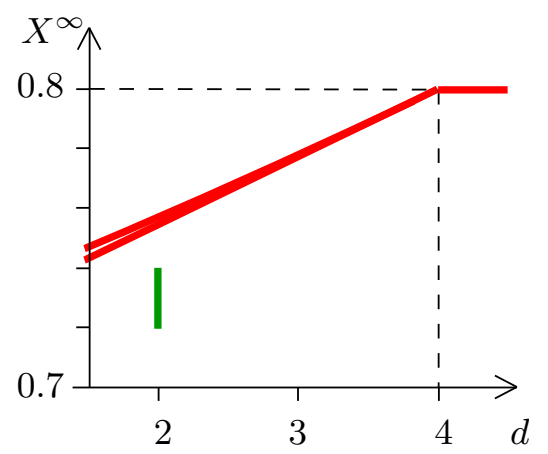

(b)

Fig. 3. Asymptotic fluctuation-dissipation ratio $X^{\infty}$ (see Eqs. (1) and (2)) as a function of the space dimensionality $d$, within the Ising universality class with purely relaxational dynamics after a quench from a disorder (a) and magnetized (b) initial state. The solid lines correspond to different Padé approximants of the two- (a) and one-loop (b) series obtained via the fieldtheoretical dimensional expansion $[14,19]$. For $d>4$ mean-field results become exact. The available numerical estimates obtained via Monte Carlo simulations $[9,14,20]$ are also indicated with the corresponding errorbars, if provided. The actual agreement between analytical predictions and numerical results is quite good.

in equation (6), with the major difference that the nonequilibrium exponent $\theta$ has been replaced by the combination $-\beta \delta /(\nu z)$ of equilibrium static and dynamic exponents. Also in this case $X^{\infty}$ can be expressed as an amplitude ratio $X^{\infty}=a_{R} /\left\{[1+\beta \delta /(\nu z)] a_{C}\right\}$ where $a_{C}$ is the non-universal constant in the scaling form of $C$ which corresponds to $a_{R}$. According to equations (6) and (7), as soon as the initial value $M_{0}$ of the magnetization is nonvanishing (quench from point B in Fig. 1), the asymptotic behaviour for large times (and therefore the value of $X^{\infty}$ ) changes compared to the case in which the initial value of the magnetization is zero (quench from point A in Fig. 1). An analogous conclusion has been drawn for different universality classes $[14,15,18]$. The full scaling functions in equation (4) actually describe the crossover which occurs, for a finite and fixed value of the magnetization $M_{0}$ (and therefore $t_{0}$ ) when both times $s$ and $t$ increases from the case $s<t \ll t_{0}$ (described by Eq. (6)) to the asymptotic case $t_{0} \ll s<t$ (described by Eq. (7)).

The field-theoretical approach yields not only the predictions, previously discussed, for the scaling behaviour and the associated exponents but also analytic expressions for other relevant universal quantities such as the fluctuation-dissipation ratio and its asymptotic limit $X^{\infty}$. Here we focus on the specific case of the universality class of the Ising model with Glauber dynamics, although analogous predictions are available for different universality classes and dynamics (see Ref. [11] for a review). In Figure 3 we report the dependence of the asymptotic fluctuation-dissipation ratio $X^{\infty}$ (see Eqs. (1) and (2)) on the space dimensionality $d$ of the model, after a quench from an initial state with $M_{0}=0$ (a) and $M_{0} \neq 0$ (b) to the critical point. The solid lines correspond to different Padé approximants of the $O\left(\epsilon^{2}\right)$ (a) [19] and $O(\epsilon)$ (b) [14] series which have been calculated within the field-theoretical $\epsilon$-expansion, where $\epsilon=4-d$. For $d>4$ the mean-field predictions $X^{\infty}\left(M_{0}=0\right)=1 / 2$ and $X^{\infty}\left(M_{0} \neq 0\right)=4 / 5$ become exact. The available Monte Carlo estimates in $d=2,3,4[9,20]$ for $M_{0}=0$ and in $d=2$ for $M_{0} \neq 0$ [14], reported in Figures 3a and 3b, respectively, are in quite good quantitative agreement with the analytical predictions.

In summary, field-theoretical methods are a viable approach to investigate non-equilibrium and ageing phenomena during critical relaxation in the bulk. The long-time properties of the non-equilibrium relaxation turn out to depend on the initial condition (e.g., $T_{0}$ ) via the corresponding magnetization $M_{0}$ and novel crossovers in the response, correlation functions and persistence properties of $M(t)$ [16] occur for finite $M_{0}$, as confirmed by Monte Carlo simulations. Within this approach one can also investigate the dependence of $X^{\infty}$ - and therefore of the effective temperature $T_{\text {eff }}=T / X^{\infty}$ - on the observable used for its definition. Interestingly enough, one finds [10] that $X^{\infty}$ (equivalently, $T_{\text {eff }}$ ) is independent of it only if the fluctuations in the system are of Gaussian nature (as in mean-field or spherical models). This observation might be of relevance to the case of more complex glassy systems.

In addition to the case illustrated in this Section, a variety of different universality classes and dynamics, both in the bulk and close to surfaces of semi-infinite systems has been investigated in the literature [11,21].

\section{Relaxation in confined geometries}

In Section 2 we discussed some aspects of critical relaxation in bulk systems, with particular focus on the nonequilibrium character of such a dynamics and on quantitative predictions for the Ising universality class with purely relaxational dynamics. In this Section we consider, instead, relaxation phenomena in confined geometries, focussing on fluctuation-induced forces and on the way they build up in time after a thermal quench. Perhaps, the most widely known example of such forces is the Casimir effect in quantum electrodynamics (QED) [22]; less known is the analogous effect which occurs in statistical physics, discussed for the first time by Fisher and de Gennes [23]. 
Indeed, whenever one confines a medium in which fluctuations (of different physical nature) take place, effective forces arise on the confining walls. The medium can be constitued by, e.g., ${ }^{4} \mathrm{He},{ }^{4} \mathrm{He} /{ }^{3} \mathrm{He}$ mixtures, classical binary mixtures, a Bose gas or even a magnetic material. At variance with $Q E D$, in statistical physics such effective forces have generically a finite range which is related to the typical correlation length $\xi$ of the confined fluctuations. When this correlation length becomes much larger than the typical microscopic length scale $\ell_{\text {micr }}$ in the system, the Casimir-like force $F_{C}$ acting on the confining walls takes a universal form

$$
\frac{F_{C}}{A}=\frac{k_{\mathrm{B}} T}{L^{d}} \vartheta(L / \xi)
$$

where $A$ is the large transverse area of the $d$-1-dimensional walls, assumed to be parallel and separated by a distance $L . \vartheta$ is a universal scaling function and therefore it is actually determined by the gross features of: (i) the system in the bulk and of the fluctuating order parameter, as mentioned in the Introduction, (ii) the gross features of the surfaces, which - to some extent - are summarized by the boundary conditions they impose on the order parameter $\varphi(\mathbf{x}, t)$ (see, e.g., Ref. [24] for a review) and (iii) the geometry of the boundaries. In what follows we shall focus on the case of parallel and infinite confining walls, i.e., on the film geometry. Due to universality, any minimal model with the same gross features as the confined systems we are interested in captures its universal behaviour, including the Casimir force $F_{C}$. This model is usually specified in terms of an effective Hamiltonian $\mathcal{H}[\varphi]$ which determines the equilibrium distribution function $P_{\text {eq }}[\varphi] \propto \exp \left\{-\mathcal{H}[\varphi] /\left(k_{\mathrm{B}} T\right)\right\}$ of the order parameter and therefore the effective free energy $\mathcal{F}$ of the model. In turn, close to the bulk critical temperature $T_{c}$ and for a system large enough (i.e., $\left.L \gg \ell_{\text {micr }}\right), \mathcal{F}$ decomposes as the sum of a bulk term proportional to the volume $V=A \times L$ of the system, one proportional to its surface area $A$ and a third one which yields the leading and universal finite-size correction we are interested in:

$$
\begin{aligned}
\mathcal{F}= & k_{\mathrm{B}} T_{c} A \\
& \times\left[L f_{\text {bulk }}(T)+f_{\text {surf }}(T)+\frac{1}{L^{d-1}} \Theta(L / \xi)+\ldots\right],
\end{aligned}
$$

where higher-order terms in $L^{-(d-1)} \times\left(\ell_{\text {micr }} / L\right)$ have been neglected. As a consequence of this decomposition, the force $F=-\delta \mathcal{F} / \delta L$ acting on the confining walls is the sum of a bulk term $F_{\text {bulk }}$ and the Casimir force $F_{C}$ which is actually the force acting on the confining walls of the film when they are "immersed" in the fluctuating medium (so that the contribution $F_{\text {bulk }}$ acting on the two opposite sides of each wall cancels, see Fig. 4). Note that this way of determining the Casimir force $F_{C}$ via the equilibrium free energy $\mathcal{F}$ gives no information about the force at a given point in space. A different approach which, in principle, provides such an information is based on the stress tensor $T_{\alpha \beta}(\mathbf{x}) . T_{\alpha \beta}(\mathbf{x})$ is a local functional of the order parameter field $\varphi(\mathbf{x})$, it is determined - in equilibrium - on the

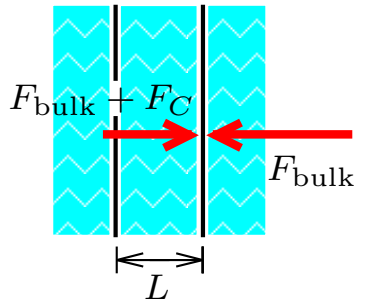

Fig. 4. Fluctuation-induced forces acting on two parallel walls immersed in a fluctuating medium. The net force on each wall is given by the Casimir term $F_{C}$.

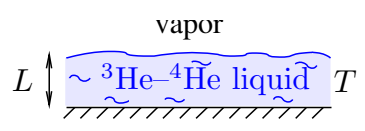

(a)

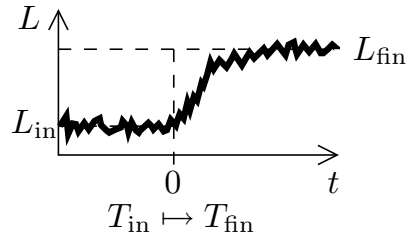

(b)
Fig. 5. (a) The equilibrium thickness $L$ of a wetting layer of a ${ }^{3} \mathrm{He} /{ }^{4} \mathrm{He}$ mixture is affected by the Casimir force, as experimentally demonstrated in reference [26]. (b) After a sudden change of the temperature from the initial value $T_{\text {in }}$ to $T_{\text {fin }}$, the Casimir force is expected to change in time so that the film, originally of thickness $L_{\mathrm{in}}$, attains its final thickness $L_{\mathrm{fin}}$ after a relaxation.

basis of $\mathcal{H}$, and its expectation value on the equilibrium distribution function $P_{\text {eq }}$ yields the force

$$
F=k_{\mathrm{B}} T A\left\langle T_{\perp \perp}(\mathbf{x})\right\rangle_{\mathrm{eq}},
$$

where $T_{\perp \perp}$ is a suitable component of the stress tensor $T_{\alpha \beta}$ (see Ref. [25] for details). In equilibrium such an average is actually independent of the position $\mathbf{x}$ at which it is calculated; nonetheless it is tempting to interpret $T_{\perp \perp}(\mathbf{x})$ as providing the local fluctuating pressure (per $k_{\mathrm{B}} T$ ) at the point $\mathbf{x}$ in space. Summing up, the equilibrium value of $F_{C}$ can be determined via both the free energy $\mathcal{F}$ and the stress tensor $T_{\alpha \beta}(\mathbf{x})$. On the other hand, if one is interested in relaxation phenomena the definition via $\mathcal{F}$ is no longer viable. Before discussing this point let us illustrate a simple experimentally relevant setting in which the relaxation of $F_{C}$ might be observed. Indeed, consider the case in which the Casimir force, in competition with dispersion forces, determines the equilibrium thickness $L$ of a wetting layer of a binary ${ }^{3} \mathrm{He} /{ }^{4} \mathrm{He}$ mixture at a given temperature $T$ (see, e.g., Refs. [26,27]), as in Figure 5a. Measuring $L$ as a function of $T$ allows a determination of the equilibrium Casimir force. Upon changing suddenly the temperature (and therefore the correlation length $\xi$ of the concentration fluctuations in the mixture) the Casimir force $F_{C}$ changes and the thickness of the layer relaxes towards its final value (as sketched in Fig. 5b). Although experimental data for the evolution of $L$ are not currently available, some aspects of the dynamics of fluctuation-induced forces are expected to be accessible in the near future. The evolution of $L$ is coupled to that one of the order parameter field (and other relevant hydrodynamic quantities) which 
evolves in time under the effect of thermal fluctuations; in turn, due to universality, this dynamics can be studied, close to critical points, via simplified models (typically in the form of Langevin evolution equations for the order parameter), as already mentioned in Section 2 (see Ref. [2] for review). Instead of discussing the problem within the experimental setup described above, in which $L$ changes in time, we assume that the fluctuating medium is confined between two parallel walls at fixed distance $L$ while the force $F_{C}(t)$ acting on them is measured (Fig. 4). As in the case of static behaviour, the presence of the confining walls imposes boundary conditions on the time-dependent order parameter field $\varphi(\mathbf{x}, t)$, the dynamics of which is affected by the confinement. In order to determine the dynamics of the Casimir force one has to understand how the local order parameter translates into the local force which can be measured, say, at the walls. One natural connection between the two is provided by the local stress which, via the time-dependence of the order parameter, becomes time dependent: $\left.T_{\alpha \beta}(\mathbf{x}, t) \equiv T_{\alpha \beta}\right|_{\varphi(\mathbf{x}) \mapsto \varphi(\mathbf{x}, t)}$. Accordingly, it is natural to define the dynamic force acting on the walls $W$ as [28] (compare with Eq. (10))

$$
F(t) \equiv k_{\mathrm{B}} T A\left\langle T_{\perp \perp}(\mathbf{x} \in W, t)\right\rangle_{\text {noise }}
$$

where the average $\langle\ldots\rangle_{\text {noise }}$ is taken over the possible realization of the thermal noise. Heuristically, this amounts to the assumption that at each time there is an "energy cost" $k_{\mathrm{B}} T A \delta L\left\langle T_{\perp \perp}\right\rangle$ associated with the displacement $\delta L$ of one of the confining wall, which is actually determined, as in equilibrium, by the order parameter field. Note that in thermal equilibrium this definition renders the static force. (See Ref. [29] for some proposed definitions of fluctuationinduced forces in different non-equilibrium system.) At variance with the equilibrium case, however, the local pressure $F / A$ might actually depend on the point $\mathbf{x}$ at which it is measured. In what follows we assume translational invariance in the direction parallel to the walls, yielding a spatially constant $F$ on each of them. Finally, in order to obtain the dynamic Casimir force $F_{C}(t)$ one has to subtract from $F(t)$ the corresponding bulk contribution $F_{\text {bulk }}(t)=\lim _{L \rightarrow \infty} F(t)$. To see this definition at work we consider the case in which $T_{\text {in }} \gg T_{c}$, corresponding to a correlation length so small that each of the walls cannot feel the effect of the confinement imposed by the other, resulting in $F_{C}(t<0)=0$. For the final temperature, instead, we assume $T_{\text {fin }}=T_{c}$, which eventually leads to a non-vanishing, long-ranged Casimir force $F_{C}(t>0) \neq 0$. (Note that, due to the critical-point shift in the film geometry [30], $T_{c}$ is not the critical temperature of the film.) The analytic results illustrated below refer to the case in which the confining walls impose Dirichlet (D: $\varphi(\mathbf{x} \in W, t)=0)$ or Neumann $\left(\mathrm{N}: \partial_{\perp} \varphi(\mathbf{x} \in W, t)=0\right.$ where $\partial_{\perp}$ is the derivative in the direction normal to the wall) boundary conditions onto the order parameter field $\varphi$. We assume that the effective Hamiltonian $\mathcal{H}$ which captures the equilibrium properties of the system is quadratic in the order parameter (Gaussian model) and that the dynamics is purely dissipative (Model A). (We point out, however, that the actual dynamic of fluids and binary mixtures requires accounting for features which are not present in Model A [2]). As a consequence of the quadratic Hamiltonian, the relevant component of the stress tensor can be expressed, in terms of the order parameter field, as $T_{\perp \perp}(\mathbf{x}, t)=\left(\partial_{\perp} \varphi(\mathbf{x}, t)\right)^{2} / 2-\left(\nabla_{\|} \varphi(\mathbf{x}, t)\right)^{2} / 2$ where $\nabla_{\|}$is the gradient parallel to the wall (see, e.g., Ref. [25]).

In the long-time limit the film attains equilibrium and the force takes different values depending on the combination of boundary conditions imposed by the two confining walls:

$$
\frac{F_{C}}{A}=\frac{k_{\mathrm{B}} T_{c}}{L^{d}} \times\left\{\begin{array}{l}
\Delta_{d}^{(\mathrm{s})}<0, \text { for } \mathrm{DD}, \mathrm{NN} \\
\Delta_{d}^{(\mathrm{ns})}>0, \text { for } \mathrm{DN}, \mathrm{ND}
\end{array}\right.
$$

where, in three dimensions $d=3$, the amplitudes for symmetric and non-symmetric boundary conditions are $\Delta_{3}^{(\mathrm{s})}=-0.048 \ldots$ and $\Delta_{3}^{(\mathrm{ns})}=+0.036 \ldots$, respectively (see, e.g., Ref. [25]). According to equation (12), the Casimir force leads to attraction between the confining walls if they impose the same boundary conditions on the fluctuating order parameter (NN, DD), whereas repulsion results from different boundary conditions (DN, $\mathrm{ND}$ ). Note that in the latter case the equilibrium force $F_{C}$ acting on the two walls is the same, independently of the fact that they impose different boundary conditions on the field; in this sense they cannot be distinguished by measuring $F_{C}$.

In view of dynamical scaling, the relaxation of the force acting on the confining walls after the thermal quench is expected to depend on the temporal scaling variable $\hat{t}=$ $\left(t / \tau_{0}\right)\left(\xi_{0} / L\right)^{z}$ via the dynamical scaling function $\vartheta_{R}(\hat{t})$,

$$
\frac{F_{C}(t)}{A}=\frac{k_{\mathrm{B}} T_{c}}{L^{d}} \vartheta_{R}\left(\hat{t}=\frac{t}{\tau_{0}}\left(\frac{\xi_{0}}{L}\right)^{z}\right),
$$

where $z=2$ is the dynamical exponent of the model we are focussing on. At small and large times we recover the initial vanishing force and its final equilibrium value in equation (12), respectively, yielding $\vartheta_{R}(0)=0$ and $\vartheta_{R}(\hat{t} \rightarrow \infty)=\Delta_{d}^{(\mathrm{s}, \mathrm{ns})}$, depending on the actual boundary conditions. In Figure 6 we report the analytic predictions for $\vartheta_{R}(\hat{t})$ (normalized to the corresponding equilibrium value $\left.\vartheta_{R}(\hat{t} \rightarrow \infty)\right)$ for the case of symmetric DD, NN (a) and non-symmetric DN, ND (b) boundary conditions. The approach to the asymptotic value depends on the boundary conditions but whereas in (a) it is independent of the wall at which $F_{C}$ is measured (due to the symmetry), this is no longer the case in (b). Accordingly, the dynamics of $F_{C}$ provides a way of determining which one of the two walls imposes D or $\mathrm{N}$ boundary condition in the DN (or ND) case, even though this is not possible by simply looking at the equilibrium force. Interestingly enough, the time dependence is not monotonic in the case of the $\mathrm{D}$ wall.

The definition (11) allows one to study the effect of an external perturbation on the Casimir force acting on the confining walls, as discussed in detail in reference [28] within the present model and DD boundary conditions. In particular, if the external field (conjugate to the order parameter) is localized in time and space, as indicated by 


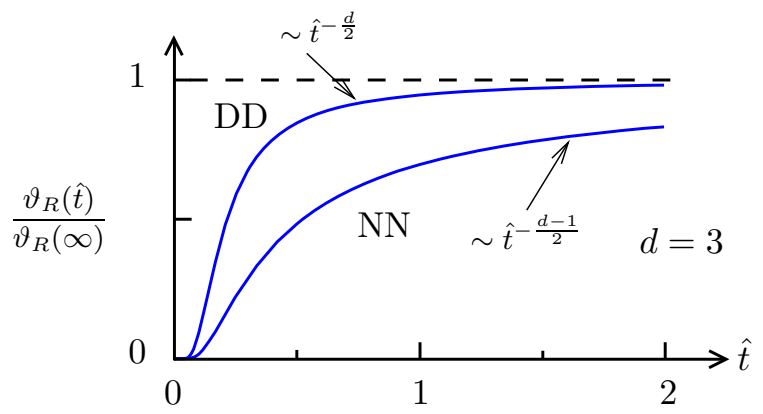

(a)

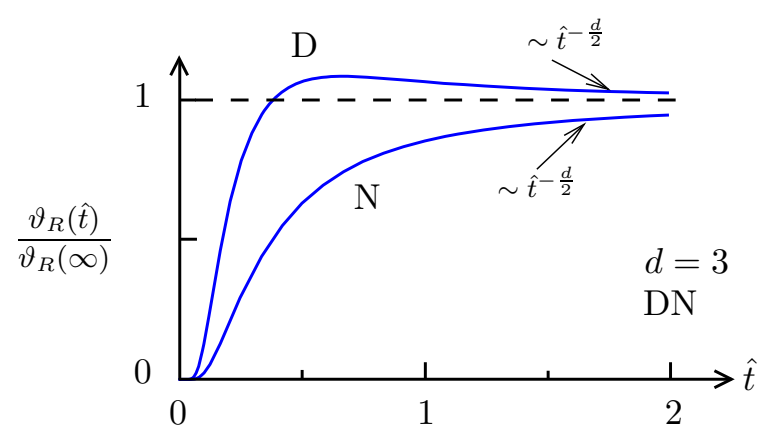

(b)

Fig. 6. Universal scaling function $\vartheta_{R}(\hat{t})$ of the Casimir force $F_{C}(t)$ in $d=3$ after a quench from the high-temperature phase to the critical point (see Eq. (13)) in the Gaussian model with purely relaxational dynamics. The finite-size scaling variable associated with time is $\hat{t}=\left(t / \tau_{0}\right)\left(\xi_{0} / L\right)^{z}$. The scaling function $\vartheta_{R}$ has been normalized by its asymptotic (equilibrium) value $\vartheta_{R}(\hat{t} \rightarrow \infty)$ (see Eq. (12)). (a) $\vartheta_{R}(\hat{t})$ for symmetric boundary conditions (DD and NN), leading to an attractive Casimir force which is equal on both the confining walls. (b) $\vartheta_{R}(\hat{t})$ for the case in which the walls impose different boundary conditions on the order parameter (DN or ND). In contrast to equilibrium, the dynamic value and the qualitative features of the time dependence of $F_{C}$ depend on the boundary condition imposed by the wall at which $F_{C}$ is measured.

the black dot in the side view in Figure $7 \mathrm{a}$, the response starts to propagate in the film, yielding asymmetric effects. At the very early stages, the response has practically not yet reached the confining walls so that the force acting on them is basically the equilibrium one corresponding to a vanishing external field. In course of time the perturbation induced by the field hits the confining walls and correspondingly the force exerted on them increases (reducing the equilibrium attraction), with different magnitude at different points. Finally, because of the relaxation character of the dynamics, the perturbation induced by the external field vanishes in the limit of long times and the effective force reaches again its equilibrium value. In Figure $7 \mathrm{~b}$ we report a sketch of the distribution of the resulting stress on the confining wall at time $\hat{t}=0.096$ in the case in which the impulse was applied at time $\hat{t}=0$ in the position indicated in Figure 7a. The system is assumed to be at the bulk critical temperature $T_{c}$. Due to the different distances from the two walls, the maximum force for $x=0$ is attained earlier on the left wall compared to the right one. (We refer the reader to Ref. [28] for further details.)

In summary, we have proposed a definition of the $d y$ namic Casimir force $F_{C}$ via the local and time-dependent stress tensor $T_{\alpha \beta}$ (see Eq. (11)), which allows one to study the equilibrium and non-equilibrium dynamics of $F_{C}$ in a variety of different cases, dynamics and boundary conditions. The analysis of very simple models reveals already a quite rich behaviour. However, it is desirable to establish a clearer connection between this definition of the dynamic Casimir force and the force that can be measured directly in actual experiments and molecular dynamics simulations.

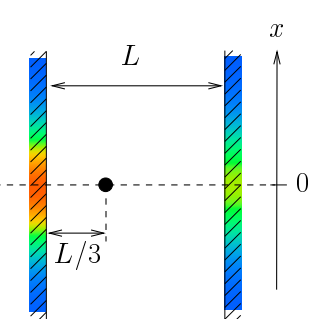

(a)

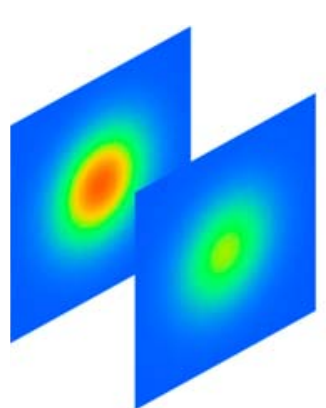

(b)
Fig. 7. Effect of an external field on the Casimir force acting on the confining walls. The impulse field is applied at time $\hat{t}=0$ and distance $L / 3$ from the left wall (a). The response of the medium is such that the Casimir force locally increases compared to its (negative) equilibrium value, reaches a maximum and then, due to the relaxational character of the dynamics, decreases again to its equilibrium value. The spatial dependence of the total local force at time $\hat{t}=0.096$ for the critical Gaussian model with DD boundary conditions is sketched in (b), where the colorcode indicates increasing force from bottom to top, compared to the equilibrium value. The magnitude of the field-induced effect depends on the square of the strength of the applied perturbation (see Ref. [28] for details).

\section{Perspectives}

In the previous sections we have reviewed some aspects of relaxation phenomena in bulk and confined critical systems. Here we mention some issues which in our opinion deserve further investigation.

It is well known that surfaces affect locally both the static and dynamic critical behaviour [24,31] and therefore we expect that also non-equilibrium properties such as the scaling behavior of two-time response and correlation functions after a quench are accordingly modified. In spite of some available Monte Carlo data and preliminary 
analyses [11,32], a detailed and quantitative study within the field-theoretical approach is still lacking. The slow collective relaxation phenomena discussed in Section 2 are due to the divergence of the equilibration time upon approaching an equilibrium critical point (characterized, e.g., by detailed balance). A qualitatively similar behaviour, however, has to be expected also in the cases in which the system undergoes a non-equilibrium phase transition of dynamical nature (e.g., reaction-diffusion systems, see Ref. [33] and references therein). Also in this case, field-theoretical methods provide valuable insight into the problem [34] and it would be desirable to extend the analysis to other relevant non-equilibrium universality classes. A particularly intriguing question is how (and if) the non-equilibrium critical relaxation phenomena in the bulk change when quantum fluctuations come into play, e.g., upon approaching a quantum phase transition point.

The study of the dynamics of fluctuation-induced forces is still far from being satisfactory and complete. In order to allow a comparison with experimentally relevant settings (e.g., involving colloidal particles in suspension) it is particularly important and urgent to explore the effects of geometries and boundary conditions different from those discussed here and to consider dynamics which are more suitable for the description of binary fluid mixtures, on which most of the experiments are based.

I am grateful to P. Calabrese, S. Dietrich, F. Krzakala, A. Maciołek, R. Paul and G. Schehr for the stimulating collaborations which lead to some of the results presented here.

\section{References}

1. J. Zinn-Justin, Quantum Field Theory and Critical Phenomena, 3rd edn. (Clarendon, Oxford 1996)

2. P.C. Hohenberg, B.I. Halperin, Rev. Mod. Phys. 49, 435 (1977)

3. A.J. Bray, Adv. Phys. 43, 357 (1994); A.J. Bray, Adv. Phys. 51, 481 (2002)

4. Z. Rácz, Phys. Rev. B 13, 263 (1976); M.E. Fisher, Z. Rácz, Phys. Rev. B 13, 5039 (1976)

5. R. Bausch, H.K. Janssen, Z. Phys. B 25, 275 (1976) ; R. Bausch, E. Eisenriegler, H.K. Janssen, Z. Phys. B 36, 179 (1979)

6. L.F. Cugliandolo, J. Kurchan, G. Parisi, J. Phys. I France 4, 1641 (1994)

7. L.F. Cugliandolo, Slow relaxation and nonequilibrium dynamics in condensed matter, Les Houches, École d'Été de Physique Théorique, Vol. 77 edited by. J-L Barrat et al. (Springer, Berlin 2003), p. 371

8. L.F. Cugliandolo, J. Kurchan, Phys. Rev. Lett. 71, 173 (1993); L.F. Cugliandolo, J. Kurchan, J. Phys. A: Math. Gen. 27, 5749 (1994); L.F. Cugliandolo, J. Kurchan, Phil. Mag. B 71, 50 (1995)

9. C. Godrèche, J.M. Luck, J. Phys. A: Math. Gen. 33, 9141 (2000); C. Godrèche, J.M. Luck, J. Phys.: Condens. Matter 14, 1589 (2002)
10. P. Calabrese, A. Gambassi, J. Stat. Mech.: Theor. Exp. P07013, (2004)

11. P. Calabrese, A. Gambassi, J. Phys. A: Math. Gen. 38, R133 (2005)

12. P. Calabrese, A. Gambassi, Phys. Rev. E 65, 066120 (2002); P. Calabrese, A. Gambassi, Acta Phys. Slov. 52, 335 (2002)

13. A. Gambassi, J. Phys.: Conf. Series 40, 13 (2006)

14. P. Calabrese, A. Gambassi, F. Krzakala, J. Stat. Mech.: Theor. Exp. P06016 (2006)

15. P. Calabrese, A. Gambassi, J. Stat. Mech.: Theor. Exp. P01001, (2007)

16. R. Paul, A. Gambassi, G. Schehr, Europhys. Lett. 78, 10007 (2007)

17. H.K. Janssen, B. Schaub, B. Schmittmann, Z. Phys. B 73, 539 (1989); H.K. Janssen, in From Phase Transitions to Chaos- Topics in Modern Statistical Physics, edited by G. Györgyi et al. (World Scientific, Singapore 1992).

18. A.A. Fedorenko, S. Trimper, Europhys. Lett. 74, 89 (2006); A. Annibale, P. Sollich, J. Phys. A: Math. Gen. 39, 2853 (2006); A. Garriga, P. Sollich, I. Pagonabarraga, F. Ritort, Phys. Rev. E 72, 056114 (2005)

19. P. Calabrese, A. Gambassi, Phys. Rev. E 66, 066101 (2002)

20. P. Mayer, L. Berthier, J.P. Garrahan, P. Sollich, Phys. Rev. E 68, 016116 (2003); E. Lippiello, F. Corberi, M. Zannetti, Phys. Rev. E 74, 041113 (2006); see Table 1 in reference [11] for a comprehensive list of Monte Carlo studies

21. P. Calabrese, A. Gambassi, Phys. Rev. B 66, 212407 (2002); P. Calabrese, A. Gambassi, Phys. Rev. E 67, 036111 (2003)

22. H.B.G. Casimir, Proc. Kon. Nederl. Akad. Wet. B 51, 793 (1948)

23. M.E. Fisher, P.G. de Gennes, C.R. Acad. Sci. Paris Ser. B 287, 207 (1978)

24. H.W. Diehl, in Phase Transitions and Critical Phenomena, edited by C. Domb, J.L. Lebowitz, Vol. 10 (Academic Press, London 1986); H.W. Diehl, Int. J. Mod. Phys. B 11, 3503 (1997)

25. M. Krech, Casimir Effect in Critical Systems (World Scientific, Singapore 1994); J.G. Brankov, D.M. Danchev, N.S. Tonchev, Theory of Critical Phenomena in Finite-size Systems: Scaling and Quantum Effects (World Scientific, Singapore 2000)

26. R. Garcia, M.H.W. Chan, Phys. Rev. Lett. 88, 086101 (2002)

27. A. Maciołek, A. Gambassi, S. Dietrich, Phys. Rev. E 76, 031124 (2007)

28. A. Gambassi, S. Dietrich, J. Stat. Phys. 123, 929 (2006)

29. D. Bartolo, A. Ajdari, J.-B. Fournier, Phys. Rev. E 67, (2003) 061112; A. Najafi, R. Golestanian, arXiv: cond-mat/0308373v1 (2003). Part of this work has been published in Europhys. Lett. 68, 776 (2004); R. Brito, U. Marini Bettolo Marconi, R. Soto, Phys. Rev. E 76, 011113 (2007)

30. H. Nakanishi, M.E. Fisher, J. Chem. Phys. 78, 3279 (1983)

31. M. Pleimling, J. Phys. A: Math. Gen. 37, R79 (2004)

32. M. Pleimling, Phys. Rev. B 70, 104401 (2004); F. Baumann, M. Pleimling, J. Phys. A: Math. Gen. 39, 1981 (2006); M. Pleimling, Phys. Rev. B 76, 104422 (2007)

33. M. Henkel, J. Phys.: Condens. Matter 19, 065101 (2007)

34. F. Baumann, A. Gambassi, J. Stat. Mech.: Theor. Exp. P01002 (2007) 